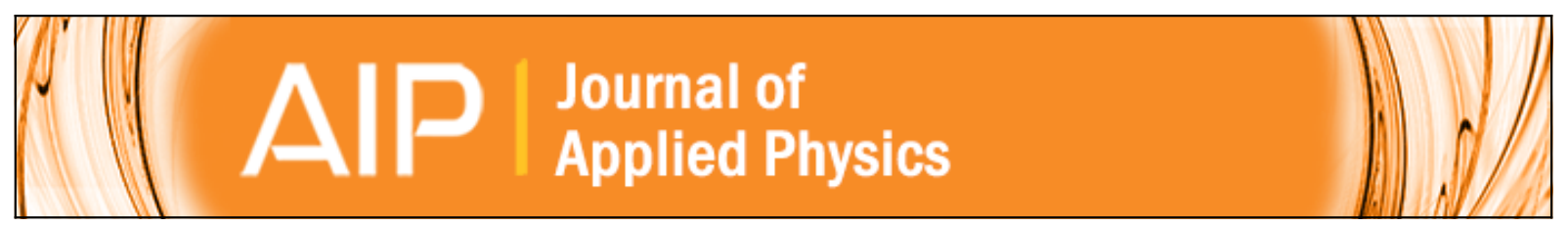

\title{
Lattice dynamics of a mist-chemical vapor deposition-grown corundum-like Ga2O3 single crystal
}

R. Cuscó, N. Domènech-Amador, T. Hatakeyama, T. Yamaguchi, T. Honda, and L. Artús

Citation: Journal of Applied Physics 117, 185706 (2015); doi: 10.1063/1.4921060

View online: http://dx.doi.org/10.1063/1.4921060

View Table of Contents: http://scitation.aip.org/content/aip/journal/jap/117/18?ver=pdfcov

Published by the AIP Publishing

\section{Articles you may be interested in}

Free-standing semipolar III-nitride quantum well structures grown on chemical vapor deposited graphene layers Appl. Phys. Lett. 103, 181108 (2013); 10.1063/1.4827539

GaN epitaxial films grown by hydride vapor phase epitaxy on polycrystalline chemical vapor deposition diamond substrates using surface nanostructuring with TiN or anodic Al oxide

J. Vac. Sci. Technol. B 28, 1011 (2010); 10.1116/1.3488616

Optical properties of GaN grown by hydride vapor-phase epitaxy

Appl. Phys. Lett. 78, 273 (2001); 10.1063/1.1338503

Development of a low-temperature GaN chemical vapor deposition process based on a single molecular source H 2 GaN 3

Appl. Phys. Lett. 74, 883 (1999); 10.1063/1.123398

How to distinguish the Raman modes of epitaxial $\mathrm{GaN}$ with phonon features from sapphire substrate-Comments on "Optical properties of GaN film grown by metalorganic chemical vapor deposition" [J. Vac. Sci. Technol. A 14, 840 (1996)]

J. Vac. Sci. Technol. A 15, 2428 (1997); 10.1116/1.580759

\section{AIP $\mid$ APL Photonics}

APL Photonics is pleased to announce Benjamin Eggleton as its Editor-in-Chief

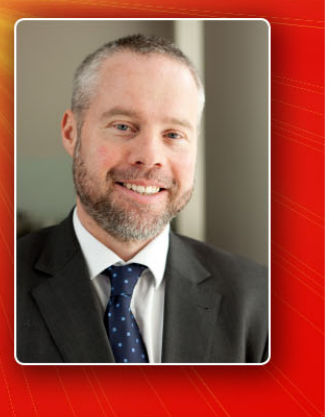




\title{
Lattice dynamics of a mist-chemical vapor deposition-grown corundum-like $\mathrm{Ga}_{2} \mathrm{O}_{3}$ single crystal
}

\author{
R. Cuscó, ${ }^{1}$ N. Domènech-Amador, ${ }^{1}$ T. Hatakeyama, ${ }^{2}$ T. Yamaguchi, ${ }^{2}$ T. Honda, ${ }^{2}$ \\ and L. Artús ${ }^{1}$ \\ ${ }^{1}$ Institut Jaume Almera (ICTJA-CSIC), Consejo Superior de Investigaciones Científicas, 08028 Barcelona, Spain \\ ${ }^{2}$ Department of Electrical Engineering and Electronics, Graduate School of Engineering, \\ Kogakuin University, 2665-1 Nakano, Hachioji, Tokyo 192-0015, Japan
}

(Received 20 March 2015; accepted 1 May 2015; published online 12 May 2015)

\begin{abstract}
The lattice dynamical properties of the corundum-like $\alpha$-phase of $\mathrm{Ga}_{2} \mathrm{O}_{3}$ are investigated by means of Raman scattering experiments and ab-initio calculations. A high-quality, single-crystal thick epilayer was grown on sapphire by the mist-chemical vapor deposition method. The phonon frequencies at the Brillouin zone center of all the Raman-active modes are determined by polarized Raman scattering measurements on an $\alpha-\mathrm{Ga}_{2} \mathrm{O}_{3}$ single crystal. By performing backscattering measurements from (0001) and (1010) faces, all Raman active modes are unambiguously identified. Density functional perturbation theory calculations were carried out to determine the symmetry and the frequency of the $\alpha-\mathrm{Ga}_{2} \mathrm{O}_{3}$ lattice modes. We find a good agreement between the theoretical predictions and the Raman spectra. The relative intensity of the different modes and their polarizability are discussed. The Raman spectrum is dominated by a narrow $A_{1 g}$ peak which indicates the high crystalline quality of the layers grown by the mist chemical vapor deposition method. (C) 2015 AIP Publishing LLC. [http://dx.doi.org/10.1063/1.4921060]
\end{abstract}

\section{INTRODUCTION}

Gallium oxide is a wide-band-gap semiconductor with important applications in transparent conductive oxides, ${ }^{1}$ deep ultraviolet solar-blind detectors, ${ }^{2,3}$ spintronics, ${ }^{4}$ high temperature gas sensors, ${ }^{5}$ and novel functional oxides. ${ }^{6}$ The monoclinic $\beta$-phase is thermodynamically the most stable phase at ambient conditions. ${ }^{7}$ Owing to its unique physical properties, such as chemical and thermal stability at high temperatures, large bandgap $\left(E_{g}=4.9 \mathrm{eV}\right)$, large breakdown field (estimated at $8 \mathrm{MV} \mathrm{cm}{ }^{-1}$ ), and high dielectric constant $(\sim 10)^{8,9}$ as well as to its widely tunable conductivity, ${ }^{10}$ a great effort has been devoted in the past decade to the development of $\beta-\mathrm{Ga}_{2} \mathrm{O}_{3}$-based device technology. $\mathrm{Ga}_{2} \mathrm{O}_{3}$ is a promising material to realize low-cost power devices outperforming those based on $\mathrm{GaN}$ or SiC. Recently, a metal semiconductor field effect transistor with good $I-V$ characteristics and a breakdown voltage over $250 \mathrm{~V}$ has been demonstrated. ${ }^{11} \mathrm{Ga}_{2} \mathrm{O}_{3}$ may also provide low-cost substrates for epitaxial growth of GaN. In spite of the dissimilarity between their crystalline structures and lattice parameters, by adjusting the nitridation conditions that transform the $\beta$ - $\mathrm{Ga}_{2} \mathrm{O}_{3}$ surface to $\mathrm{GaN}$, homoepitaxial growth of high quality hexagonal $\mathrm{GaN}$ on $\beta-\mathrm{Ga}_{2} \mathrm{O}_{3}$ was achieved by molecular beam epitaxy. ${ }^{12}$

While most of the progress in $\mathrm{Ga}_{2} \mathrm{O}_{3}$-based devices has been developed on the $\beta$-phase, the less-studied metastable, corundum-like $\alpha$-phase has also a great potentiality in the integration of transparent conductive oxide technology with other corundum structure functional oxides, such as $\mathrm{Cr}, \mathrm{Fe}$, and $\mathrm{V}$ oxides. The potential of alloying with $\alpha-\mathrm{Fe}_{2} \mathrm{O}_{3}$ and $\alpha-\mathrm{Cr}_{2} \mathrm{O}_{3}$ opens up the possibility of harnessing multiferroic and magnetoelectric effects. ${ }^{4,13}$ On the other hand, the $\alpha-\mathrm{Ga}_{2} \mathrm{O}_{3}$ has a moderate in-plane $a$-lattice parameter misfit $(\sim 4.8 \%)^{14}$ with $\alpha-\mathrm{Al}_{2} \mathrm{O}_{3}$ and a substantially lower bandgap $\left(\sim 5 \mathrm{eV} \text { for } \alpha-\mathrm{Ga}_{2} \mathrm{O}_{3}\right)^{15,16}$ compared to sapphire. This makes the $\alpha-\mathrm{Ga}_{2} \mathrm{O}_{3} / \alpha-\mathrm{Al}_{2} \mathrm{O}_{3}$ system an interesting possibility for the development of intersubband devices operating at telecommunication wavelengths. Very recently, it has been shown that three monolayers of pseudomorphic $\alpha-\mathrm{Ga}_{2} \mathrm{O}_{3}$ stabilized by strain are present at the interface between $c$-plane sapphire and $\beta-\mathrm{Ga}_{2} \mathrm{O}_{3} .{ }^{17}$

The growth of highly crystalline $\alpha-\mathrm{Ga}_{2} \mathrm{O}_{3}$ by mist chemical vapor deposition (CVD) on $c$-sapphire was demonstrated by Shinohara and Fujita. ${ }^{16}$ More recently, highly conductive $n$-type layers of tin-doped $\alpha-\mathrm{Ga}_{2} \mathrm{O}_{3}$ grown by mist CVD have been reported. ${ }^{18} \mathrm{~A}$ semi-coherent growth with the $\alpha-\mathrm{Al}_{2} \mathrm{O}_{3}$ substrate has been proposed to account for misfit relaxation at the interface and the low density of screw dislocations ${ }^{14}$ which contribute to obtain samples with excellent crystalline quality. The growth of high quality $\alpha-\mathrm{Ga}_{2} \mathrm{O}_{3}$ epilayers on sapphire is also favored by the fact that both epilayer and substrate have the same crystalline structure. The mist-CVD technique is an environmentally friendly growth method that can provide a cost-effective way of producing large-area, high-quality $\alpha-\mathrm{Ga}_{2} \mathrm{O}_{3}$ buffer layers. These could have a great potential as novel buffer layers for the growth of hexagonal GaN layers as they have the same rhombohedric crystalline structure as sapphire and an excellent lattice match of $\mathrm{GaN}$ with the nitridated $\alpha-\mathrm{Ga}_{2} \mathrm{O}_{3}$ surface is expected.

Raman scattering is a powerful, nondestructive tool to characterize the crystal quality and strain state of thin film layered materials. While the polarized Raman spectra of $\beta$ - $\mathrm{Ga}_{2} \mathrm{O}_{3}$ was reported on (010) Mg-doped, (100) Si-doped, and (001) unintentionally doped layers obtained by the floating-zone and edge-defined film-fed growth, ${ }^{19}$ no similar 
study exists on $\alpha-\mathrm{Ga}_{2} \mathrm{O}_{3}$. The only Raman-scattering data on phonons of $\alpha-\mathrm{Ga}_{2} \mathrm{O}_{3}$ were reported by Machon et al. ${ }^{7}$ in a study of $\beta$ - to $\alpha$-phase transition under hydrostatic pressure. The high-pressure form of $\mathrm{Ga}_{2} \mathrm{O}_{3}$ contains a substantial degree of structural disorder, and to help identify the features in the Raman spectrum it was compared with an unpolarized spectrum recorded on a sample obtained from a precipitated gel in aqueous solution. ${ }^{7}$ In the present work, we present polarized Raman scattering spectra obtained in backscattering configuration from (0001) and (1010) faces of a corundum-like $\alpha-\mathrm{Ga}_{2} \mathrm{O}_{3}$ monocrystalline layer grown by mist CVD. The thickness of the high-quality $\alpha-\mathrm{Ga}_{2} \mathrm{O}_{3}$ layer was high enough to allow micro-Raman measurements to be carried out on a lateral fracture face. This enabled us to verify Raman selection rules and to identify all Raman active modes in the $\alpha-\mathrm{Ga}_{2} \mathrm{O}_{3}$ crystal.

\section{EXPERIMENT}

A corundum-like $\alpha-\mathrm{Ga}_{2} \mathrm{O}_{3}$ layer of thickness around $0.8 \mu \mathrm{m}$ was grown by the ultrasonic mist chemical vapor deposition on a $c$-face sapphire substrate. A liquid solution of $\mathrm{Ga}\left(\mathrm{C}_{5} \mathrm{H}_{7} \mathrm{O}_{2}\right)_{3}$ in $\mathrm{H}_{2} \mathrm{O}(0.05 \mathrm{~mol} / \mathrm{l})$ was used as $\mathrm{Ga}$ source. Mist particles were formed by a $2.4 \mathrm{MHz}$ ultrasonic transducer and carried by a flow of $\mathrm{N}_{2}$ gas to the heated reaction chamber where the (0001) sapphire substrate was placed on a sample holder kept at $200^{\circ} \mathrm{C}$. The sample was characterized by x-ray diffraction (XRD). As can be seen in Fig. 1, the $2 \theta / \theta$ scan spectra show a distinct $(0006) \alpha-\mathrm{Ga}_{2} \mathrm{O}_{3}$ reflection peak, and the XRD rocking curve (inset of Fig. 1) displays a full width at half maximum of $69 \mathrm{arcsec}$, indicating the high crystalline quality of the sample.

Raman measurements were excited with the $532 \mathrm{~nm}$ line of a diode-pumped solid-state laser. The light was collected in backscattering geometry through a $100 \times$ objective with a numerical aperture of 0.95 and analyzed using a Jobin-Yvon T64000 spectrometer. Confocal micro-Raman measurements were performed with a $100 \mu \mathrm{m}$ pinhole aperture at the back focal plane. To aid the identification of the Raman active phonons, a theoretical investigation of the lattice dynamics of $\alpha-\mathrm{Ga}_{2} \mathrm{O}_{3}$ was carried out. The numerical calculations were

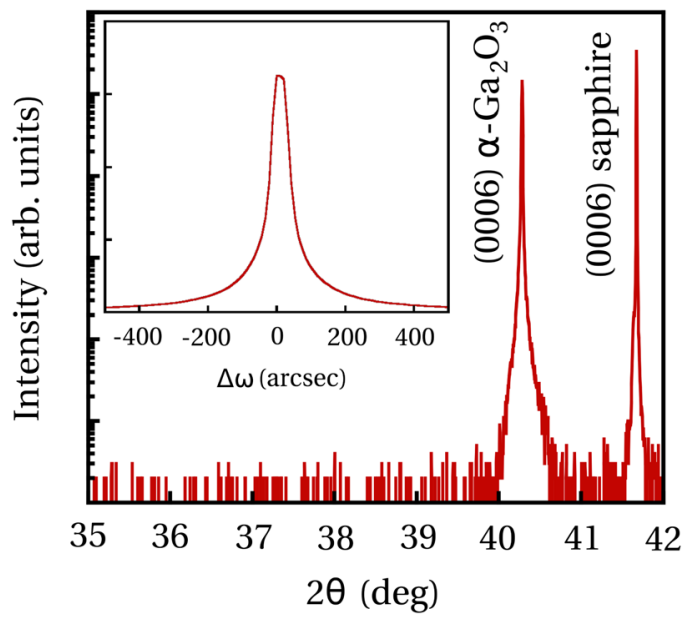

FIG. 1. X-ray diffraction 2 $2 \theta / \theta$ scan profile. Inset: (0006) XRD diffraction rocking curve. The full width at half maximum is 69 arcsec. performed in the framework of the density functional theory (DFT) in the local density approximation (LDA) as implemented in the ABINIT code. ${ }^{20}$ In the calculations, we used Perdew-Wang parametrization of the exchange interaction, Troullier-Martins pseudopotentials with the $3 d$ electrons included as valence states, a $4 \times 4 \times 4$ Monkhorst-pack $k$-point sampling and a plane-wave basis set with an energy cutoff of $95 \mathrm{Ha}^{20}$ After a full structural relaxation of the $\alpha-\mathrm{Ga}_{2} \mathrm{O}_{3}$ unit cell, hexagonal lattice parameters $a=5.004 \AA$ and $c=13.410 \AA$ were obtained, in excellent agreement with the reported high-resolution x-ray diffraction value $(a=4.983 \AA$ and $c=13.433 \AA$ ). ${ }^{21}$ Phonon modes were obtained by diagonalization of the dynamical matrix derived from the perturbation theory linear response approach. The frequencies of the zone-center Raman active phonons are listed in Table I.

\section{RESULTS AND DISCUSSION}

The $\alpha-\mathrm{Ga}_{2} \mathrm{O}_{3}$ crystal has a rhombohedral structure that belongs to the $R \overline{3} c\left(D_{3 d}^{6}\right)$ space group with two molecular $\mathrm{Ga}_{2} \mathrm{O}_{3}$ groups per unit cell. Since the $\alpha-\mathrm{Ga}_{2} \mathrm{O}_{3}$ crystal is centrosymmetric, all the Raman active phonons are infrared inactive and vice versa. The irreducible representations for the zone-center optical modes are $\Gamma=2 A_{1 g}+2 A_{1 u}+3 A_{2 g}$ $+2 A_{2 u}+5 E_{g}+4 E_{u}$. The $A_{1 g}$ and $E_{g}$ vibrations are Raman active, the $A_{2 u}$ and $E_{u}$ are infrared active, and the $A_{1 u}$ and $A_{2 g}$ have no optical activity (silent modes). The Raman tensors for the Raman active modes are

$$
\begin{aligned}
\mathcal{R}\left(A_{1 g}\right) & =\left(\begin{array}{lll}
a & 0 & 0 \\
0 & a & 0 \\
0 & 0 & b
\end{array}\right) \\
\mathcal{R}\left(E_{g}\right) & =\left(\begin{array}{ccc}
c & 0 & 0 \\
0 & -c & d \\
0 & d & 0
\end{array}\right),\left(\begin{array}{ccc}
0 & -c & -d \\
-c & 0 & 0 \\
-d & 0 & 0
\end{array}\right) .
\end{aligned}
$$

Thus, in backscattering from the $c$ face the fully symmetric $A_{1 g}$ vibrations are allowed in parallel polarization and forbidden in cross polarization, whereas the doubly degenerate $E_{g}$ vibrations are always allowed. Figure 2 shows the Raman spectra obtained in backscattering from the $c$ face. For clarity, the Raman spectrum of the sapphire substrate has been subtracted. The assignment of the symmetry of modes has been made by comparison with the DFT calculations. The $z(x x) \bar{z}$ spectrum is dominated by a strong and narrow

TABLE I. Frequencies of the Raman active modes of $\alpha-\mathrm{Ga}_{2} \mathrm{O}_{3}$.

\begin{tabular}{lcc}
\hline \hline \multirow{2}{*}{ Symmetry } & \multicolumn{2}{c}{ Phonon frequency $\left(\mathrm{cm}^{-1}\right)$} \\
\cline { 2 - 3 } & measured (Raman) & calculated (DFT) \\
\hline$A_{1 g}$ & 218.2 & 211 \\
$E_{g}$ & 240.7 & 233 \\
$E_{g}$ & 285.3 & 281 \\
$E_{g}$ & 328.8 & 314 \\
$E_{g}$ & 430.7 & 426 \\
$A_{1 g}$ & 569.7 & 551 \\
$E_{g}$ & 686.7 & 667 \\
\hline \hline
\end{tabular}




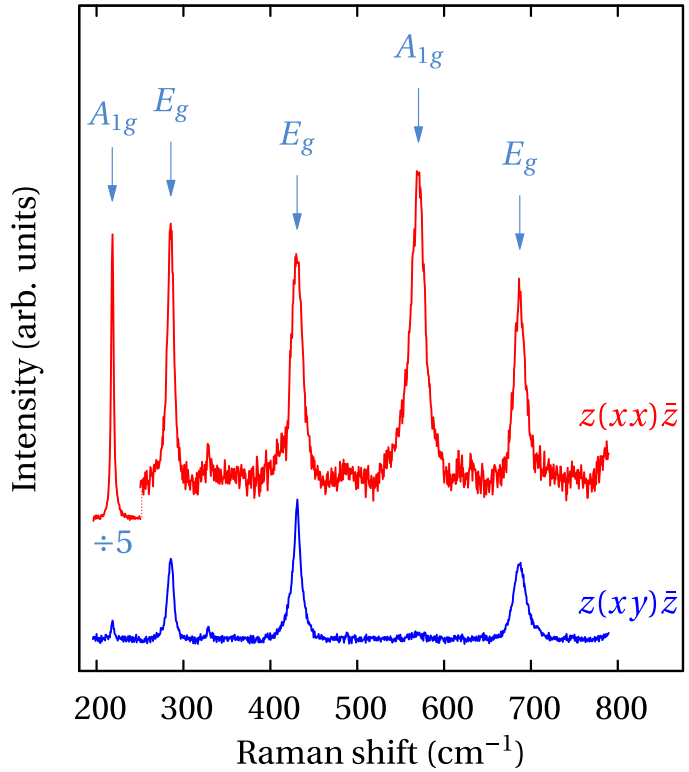

FIG. 2. Polarized Raman spectra of $\alpha-\mathrm{Ga}_{2} \mathrm{O}_{3}$ obtained in backscattering configuration from the $c$ face.

$A_{1 g}$ mode at $218.2 \mathrm{~cm}^{-1}$. This low-frequency $A_{1 g}$ mode corresponds mainly to $\mathrm{Ga}$ atoms vibrating against each other along the $c$ axis. By contrast, the high-frequency $A_{1 g}$ mode at $569.7 \mathrm{~cm}^{-1}$ involves mainly the lighter $\mathrm{O}$ atoms vibrating perpendicularly to the $c$ axis. The low-frequency $A_{1 g}$ peak exhibits the lowest linewidth (full width at half maximum of $3.3 \mathrm{~cm}^{-1}$, compared to $18.4 \mathrm{~cm}^{-1}$ for the high-frequency $A_{1 g}$ mode). The narrow linewidth of the low-frequency $A_{1 g}$ mode reflects a substantially higher phonon lifetime which can be explained in terms of the availability of anharmonic decay channels. Similar to the case of the low-frequency $E_{2}$ mode in $\mathrm{ZnO}^{22}$ and $\mathrm{InN},{ }^{23}$ the low density of phonon states with lower frequencies strongly reduces the probability of anharmonic decay into lower-energy phonons.

In addition to the $A_{1 g}$ peaks, three other Raman peaks are observed in the upper spectrum of Fig. 2, which are assigned to $E_{g}$ modes, in accordance with the DFT calculations. These $E_{g}$ modes are also present in the $z(x y) \bar{z}$ spectrum, where the symmetry forbidden $A_{1 g}$ modes are extinguished. However, no clear observation of the remaining $E_{g}$ modes (predicted at 233 and $314 \mathrm{~cm}^{-1}$ by the DFT calculations) can be made in these spectra.

To experimentally detect the remaining Raman active modes, micro Raman measurements were performed on a lateral fracture surface of the sample. While the brittle corundum structure does not have natural cleavage planes, crystals tend to part more easily in certain directions. The lowest surface energies are found for the $\{10 \overline{1} 0\}$ and $\{\overline{1} 012\}$ planes. $^{24}$ The orientation of the lateral surface in our sample was determined to be $(10 \overline{1} 0)$ by means of XRD measurements.

Figure 3 shows the polarized Raman spectra obtained from the $(10 \overline{1} 0)$ lateral face. By suitably rotating the Raman tensors [Eq. (1)] to describe the experimental scattering configuration, the Raman selection rules listed in Table II are obtained. On the $(10 \overline{1} 0)$ face, the $A_{1 g}$ modes are allowed in parallel polarization and forbidden in cross polarization. The $A_{1 g}$ low-frequency mode is again dominant in the $x^{\prime}\left(y^{\prime} y^{\prime}\right) \bar{x}^{\prime}$

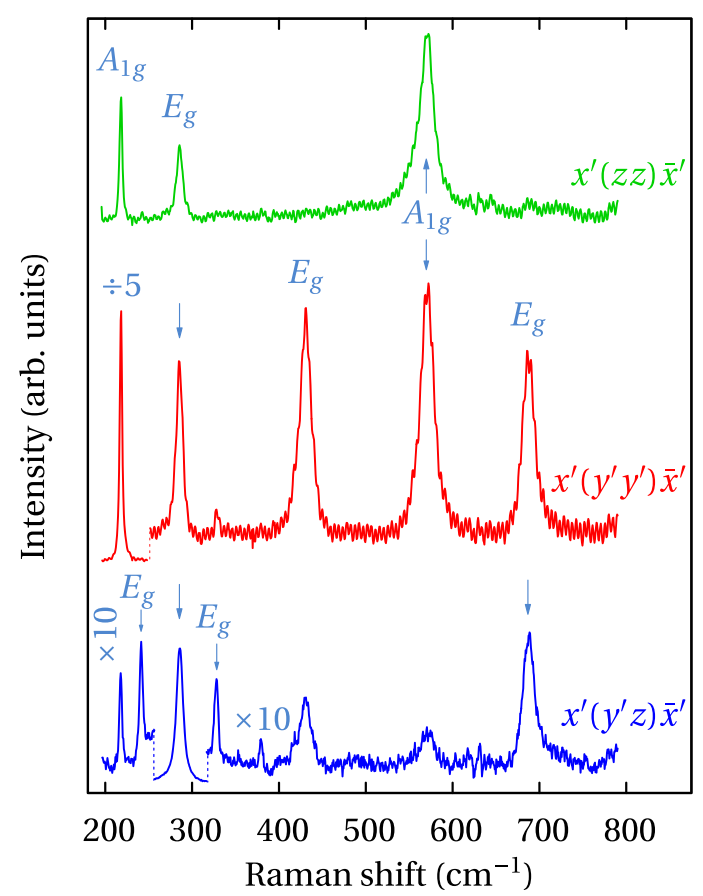

FIG. 3. Polarized Raman spectra of $\alpha-\mathrm{Ga}_{2} \mathrm{O}_{3}$ obtained in backscattering configuration from the $(10 \overline{1} 0)$ face.

spectrum. In fact, the relative intensities between Raman peaks in $x^{\prime}\left(y^{\prime} y^{\prime}\right) \bar{x}^{\prime}$ and $z(x x) \bar{z}$ spectra are very similar, as for each mode the same polarizability component is probed in both configurations (see Table II). In contrast, the intensity of the low-frequency $A_{1 g}$ mode is substantially reduced in the $x^{\prime}(z z) \bar{x}^{\prime}$ configuration, which indicates that the polarizability of this mode along the $c$-axis is significantly lower $(b \ll a)$. Even though this mode mainly involves vibrations of the $\mathrm{Ga}$ atoms against each other along the $c$ direction, the resulting radial stretching of the $\mathrm{Ga}-\mathrm{O}$ bonds yields a substantial in-plane polarizability.

Although $E_{g}$ modes are forbidden in $x^{\prime}(z z) \bar{x}^{\prime}$ configuration, a weak $E_{g}$ peak at $285.3 \mathrm{~cm}^{-1}$ is detected in the Raman spectrum. This may probably be attributed to a slight sample misalignment that introduces some leakage from the $x^{\prime}\left(y^{\prime} z\right) \bar{x}^{\prime}$ geometry. Note that the $E_{g}$ peak at $285.3 \mathrm{~cm}^{-1}$ is by far the most intense feature in the $x^{\prime}\left(y^{\prime} z\right) \bar{x}^{\prime}$ spectrum. Besides the Raman modes detected in backscattering from the $c$-face, two additional peaks are clearly seen in the $x^{\prime}\left(y^{\prime} z\right) \bar{x}^{\prime}$ spectrum at 240.7 and $328.8 \mathrm{~cm}^{-1}$ which are assigned to $E_{g}$ modes. These modes are barely visible in the $x^{\prime}\left(y^{\prime} y^{\prime}\right) \bar{x}^{\prime}$ spectrum, which implies that $c \ll d$. Conversely, the $E_{g}$ modes at 430.7 and $686.7 \mathrm{~cm}^{-1}$ display a substantially higher intensity in the $x^{\prime}\left(y^{\prime} y^{\prime}\right) \bar{x}^{\prime}$ spectrum than in the $x^{\prime}\left(y^{\prime} z\right) \bar{x}^{\prime}$ spectrum, and

TABLE II. Raman selection rules $\left(\left|\mathbf{e}_{\mathrm{i}} \mathcal{R} \mathbf{e}_{\mathrm{s}}\right|^{2}\right)$ for the different incident $\left(\mathbf{e}_{\mathrm{i}}\right)$ and scattered $\left(\mathbf{e}_{\mathrm{s}}\right)$ polarizations used in the Raman experiments. Here, $x^{\prime}=\frac{\sqrt{3}}{2} x$ $-\frac{1}{2} y$ and $y^{\prime}=\frac{1}{2} x+\frac{\sqrt{3}}{2} y$.

\begin{tabular}{lcccccc}
\hline \hline & \multirow{2}{*}{ Backscattering from (0001) face } & & \multicolumn{2}{c}{ Backscattering from (1010) face } \\
\cline { 2 - 2 } \cline { 5 - 7 } & $z(x x) \bar{z}$ & $z(x y) \bar{z}$ & & $x^{\prime}(z z) \bar{x}^{\prime}$ & $x^{\prime}\left(y^{\prime} y^{\prime}\right) \bar{x}^{\prime}$ & $x^{\prime}\left(y^{\prime} z\right) \bar{x}^{\prime}$ \\
\hline$A_{1 g}$ & $a^{2}$ & 0 & & $b^{2}$ & $a^{2}$ & 0 \\
$E_{g}$ & $c^{2}$ & $c^{2}$ & & 0 & $c^{2}$ & $d^{2}$ \\
\hline \hline
\end{tabular}


therefore for these modes we have $c \gg d$. In contrast, the $E_{g}$ mode at $285.3 \mathrm{~cm}^{-1}$ displays a similar intensity in both geometries, and consequently $c \simeq d$ for this mode.

The Raman measurements on the lateral face of the $\alpha-\mathrm{Ga}_{2} \mathrm{O}_{3}$ epilayer have revealed all Raman active modes $\left(2 A_{1 g}+5 E_{g}\right)$. Their frequencies are listed in Table I, compared with the frequencies obtained from DFT calculations. A good overall agreement is found between experimental and calculated values, although the measured Raman frequencies are systematically higher than the theoretical values by $\sim 3 \%$ on average. The presence of a compressive residual strain in the $\alpha-\mathrm{Ga}_{2} \mathrm{O}_{3}$ cannot be ruled out, given the in-plane lattice mismatch with the sapphire substrate $(\sim 4.8 \%)$. Transmission electron microscopy studies suggests semicoherent growth of $\alpha-\mathrm{Ga}_{2} \mathrm{O}_{3}$ on sapphire, with some degree of misfit relaxation at the interface giving rise to periodic structures along the [1100] direction. ${ }^{14}$ The misfit dislocations do not thread through the layer, which explain the high crystalline quality observed in these crystals by $\mathrm{x}$-ray and Raman measurements. We have not observed significant frequency shifts between samples of different thicknesses, which is consistent with the localization of the misfit defects primarily on the interface.

\section{SUMMARY AND CONCLUSIONS}

A high-quality, single crystalline layer of the corundumlike $\alpha$-phase of $\mathrm{Ga}_{2} \mathrm{O}_{3}$ has been grown by the mist-CVD method on a (0001)-sapphire substrate. The vibrational modes of $\alpha-\mathrm{Ga}_{2} \mathrm{O}_{3}$ have been studied by means of Raman scattering. The thickness of the $\alpha-\mathrm{Ga}_{2} \mathrm{O}_{3}$ layer permits micro-Raman measurements to be carried out on a $(10 \overline{1} 0)$ lateral face, which gives access to additional scattering configurations. This allowed us to observe all Raman active modes of $\alpha-\mathrm{Ga}_{2} \mathrm{O}_{3}$. Whereas both $A_{1 g}$ and $E_{g}$ modes are allowed in the customarily used backscattering geometry on the $c$ face, two of the $E_{g}$ modes are only unambiguously revealed in backscattering from a lateral face in cross polarization. The frequencies of the modes are in good agreement with DFT calculations. The polarized spectra are consistent with the Raman selection rules, indicating the high crystalline quality of the sample, and yield information about the polarizability of the different modes. The Raman spectrum is dominated by the low frequency $A_{1 g}$ mode, which corresponds mainly to $\mathrm{Ga}$ atoms vibrating against each other along the $c$ axis and displays a remarkably narrow linewidth. Raman spectroscopy can be a versatile tool for the characterization of $\alpha-\mathrm{Ga}_{2} \mathrm{O}_{3}$ layers in a new emerging device technology based on functional oxides.

\section{ACKNOWLEDGMENTS}

This work has been supported by the Spanish Ministry of Economy and Competitiveness under Contract Nos. MAT2010-16116 and MAT2014-59199. N.D. acknowledges financial support from the FPU program of the Spanish Ministry of Education. T.H. and T.Y. acknowledge financial support from the Ministry of Education, Culture, Sports, Science and Technology of Japan (MEXT) under the Strategic Research Foundation Grant-aided Project for Private Universities 2011-2015 (S1101005) and the Grants-in-Aid for Scientific Research Nos. 25420341 and 25706020, as well as from the Advanced Low Carbon Technology Research and Development Program, JST, Japan.

${ }^{1}$ N. Ueda, H. Hosono, R. Waseda, and H. Kawazoe, Appl. Phys. Lett. 70, 3561 (1997).

${ }^{2}$ T. Oshima, T. Okuno, N. Arai, N. Suzuki, S. Ohira, and S. Fujita, Appl. Phys. Express 1, 011202 (2008).

${ }^{3}$ L. Li, E. Auer, M. Liao, X. Fang, T. Zhai, U. K. Gautam, A. Lugstein, Y. Koide, Y. Bando, and D. Golberg, Nanoscale 3, 1120 (2011).

${ }^{4}$ K. Kaneko, T. Nomura, I. Kakeya, and S. Fujita, Appl. Phys. Express 2, 075501 (2009).

${ }^{5}$ U. Hoefer, J. Frank, and M. Fleischer, Sens. Actuators, B 78, 6 (2001).

${ }^{6}$ Z. Dai, Z. Pan, and Z. Wang, Adv. Funct. Mater. 13, 9 (2003).

${ }^{7}$ D. Machon, P. McMillan, B. Xu, and J. Dong, Phys. Rev. B 73, 094125 (2006).

${ }^{8}$ M. Passlack, E. F. Schubert, W. S. Hobson, M. Hong, N. Moriya, S. N. G. Chu, K. Konstadinidis, J. P. Mannaerts, M. L. Schnoes, and G. J. Zydzik, J. Appl. Phys. 77, 686 (1995).

${ }^{9}$ M. Higashiwaki, K. Sasaki, A. Kuramata, T. Masui, and S. Yamakoshi, Phys. Status Solidi A 211, 21 (2014).

${ }^{10}$ M. Orita, H. Ohta, M. Hirano, and H. Hosono, Appl. Phys. Lett. 77, 4166 (2000).

${ }^{11}$ M. Higashiwaki, K. Sasaki, A. Kuramata, T. Masui, and S. Yamakoshi, Appl. Phys. Lett. 100, 013504 (2012).

${ }^{12}$ S. Ohira, N. Suzuki, H. Minami, K. Takahashi, T. Araki, and Y. Nanishi, Phys. Status Solidi C 4, 2306 (2007).

${ }^{13}$ N. Kida, Y. Kaneko, J. He, M. Matsubara, H. Sato, T. Arima, H. Akoh, and Y. Tokura, Phys. Rev. Lett. 96, 167202 (2006).

${ }^{14}$ K. Kaneko, H. Kawanowa, H. Ito, and S. Fujita, Jpn. J. Appl. Phys., Part 1 51, 020201 (2012).

${ }^{15}$ G. Sinha, K. Adhikary, and S. Chaudhuri, J. Cryst. Growth 276, 204 (2005).

${ }^{16}$ D. Shinohara and S. Fujita, Jpn. J. Appl. Phys., Part 1 47, 7311 (2008).

${ }^{17}$ R. Schewski, G. Wagner, M. Baldini, D. Gogova, Z. Galazka, T. Schulz, T. Remmele, T. Markurt, H. von Wenckstern, M. Grundmann et al., Appl. Phys. Express 8, 011101 (2015).

${ }^{18}$ K. Akaiwa and S. Fujita, Jpn. J. Appl. Phys., Part 1 51, 070203 (2012).

${ }^{19}$ T. Onuma, S. Fujioka, T. Yamaguchi, Y. Itoh, M. Higashiwaki, K. Sasaki, T. Masui, and T. Honda, J. Cryst. Growth 401, 330 (2014).

${ }^{20}$ X. Gonze, J. M. Beuken, R. Caracas, F. Detraux, M. Fuchs, G. M. Rignanese, L. Sindic, M. Verstraete, G. Zerah, F. Jollet et al., Comput. Mater. Sci. 25, 478 (2002).

${ }^{21}$ M. Marezio and J. P. Remeika, J. Chem. Phys. 46, 1862 (1967).

${ }^{22}$ M. Millot, R. Tena-Zaera, V. Muñoz-Sanjosé, J. M. Broto, and J. González, Appl. Phys. Lett. 96, 152103 (2010).

${ }^{23}$ N. Domènech-Amador, R. Cuscó, L. Artús, T. Yamaguchi, and Y. Nanishi, Phys. Rev. B 83, 245203 (2011).

${ }^{24}$ A. Azhdari and S. Nemat-Nasser, Mech. Mater. 28, 247 (1998). 